Supporting Information

\title{
Hybrid OER Electrocatalyst Combining Mesoporous Hollow Spheres of N, P-doped Carbon with Ultrafine $\mathrm{CO}_{2} \mathrm{NiO}_{x}$
}

Jingjing Wang and Hua Chun Zeng*

Department of Chemical and Biomolecular Engineering, Faculty of Engineering, National University of Singapore, 10 Kent Ridge Crescent, Singapore 119260

*Email: chezhc@nus.edu.sg

4.

Contents

Figures S1 to S15:

Table S1 to Table S2:

References:
Pages 2 to 10

Pages 11

Pages 12 

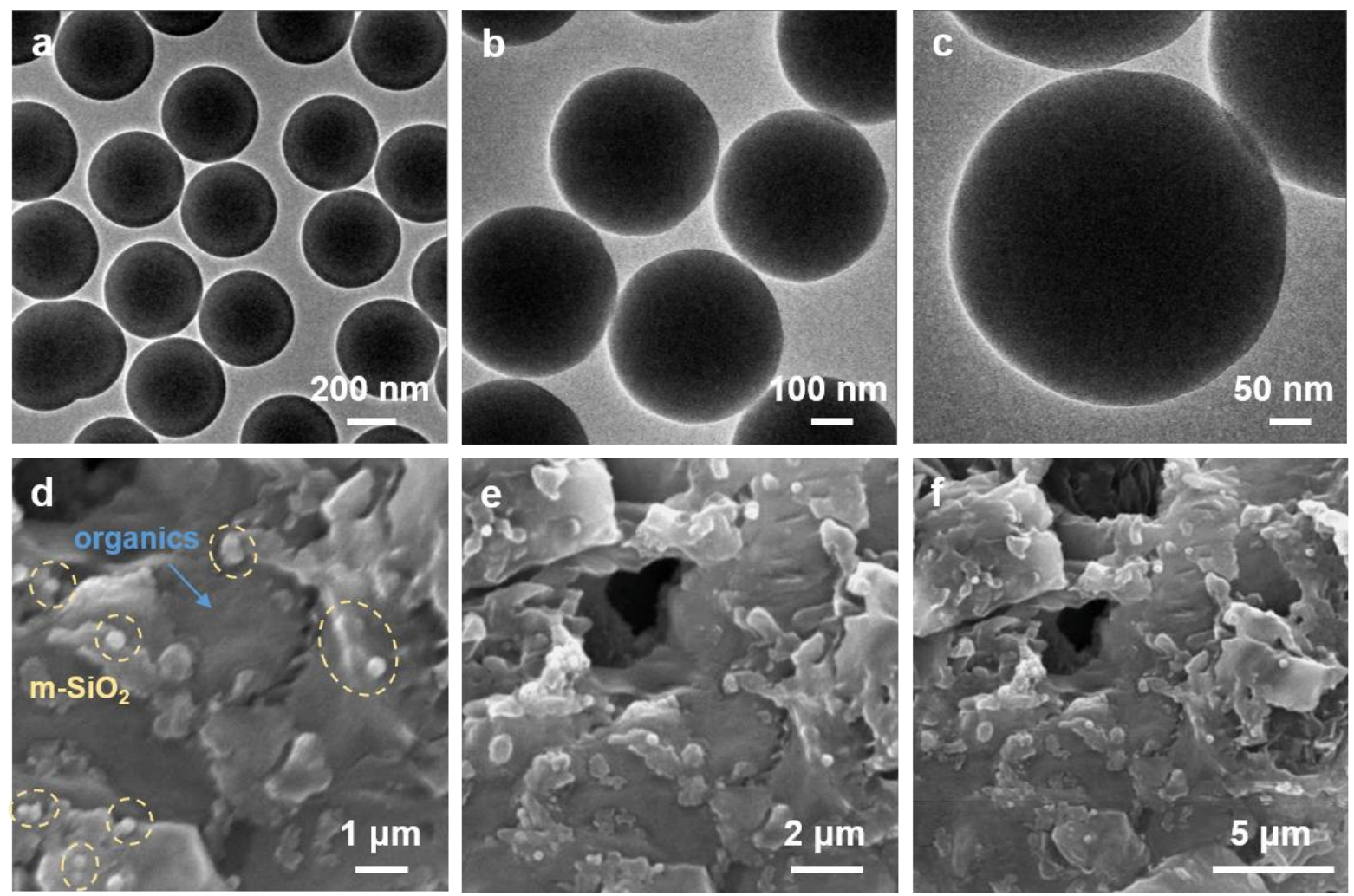

Figure S1. (a-c) TEM images at different magnifications of original $\mathrm{SiO}_{2}$ spheres. (d-f) $\mathrm{SEM}$ images at different magnifications of organics mixed with meso- $\mathrm{SiO}_{2}$ spheres with a weight ratio of 10:1.
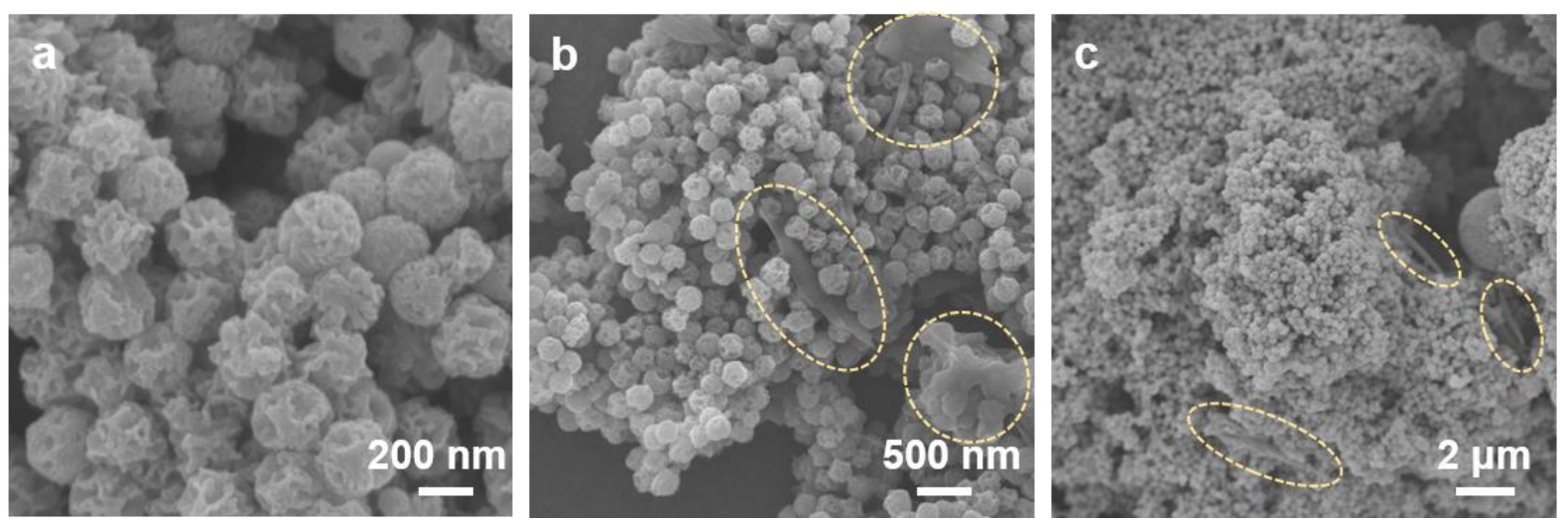

Figure S2. (a-c) SEM images at different magnifications of meso- $\mathrm{SiO}_{2} / \mathrm{NPC}$ obtained by organics filling $(10: 1)$ and pyrolysis $\left(700^{\circ} \mathrm{C}\right)$. A small amount of bulk-phase NPC occurred (marked with yellow circles) when ratio of 10 is adopted. 

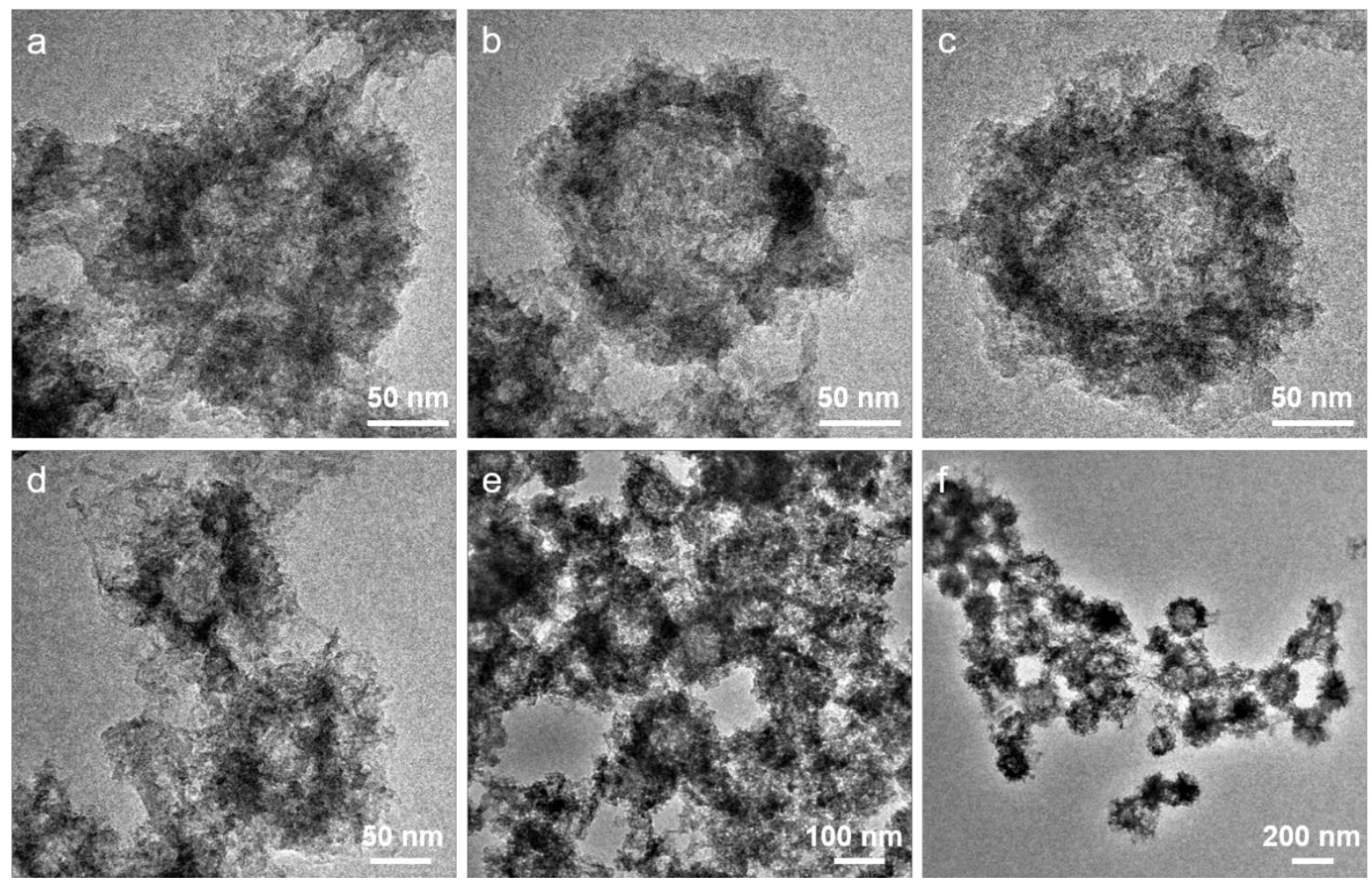

Figure S3. (a-f) TEM images at different magnifications of meso-NPC when the ratio of organics and meso- $\mathrm{SiO}_{2}$ sphere is 5:1.
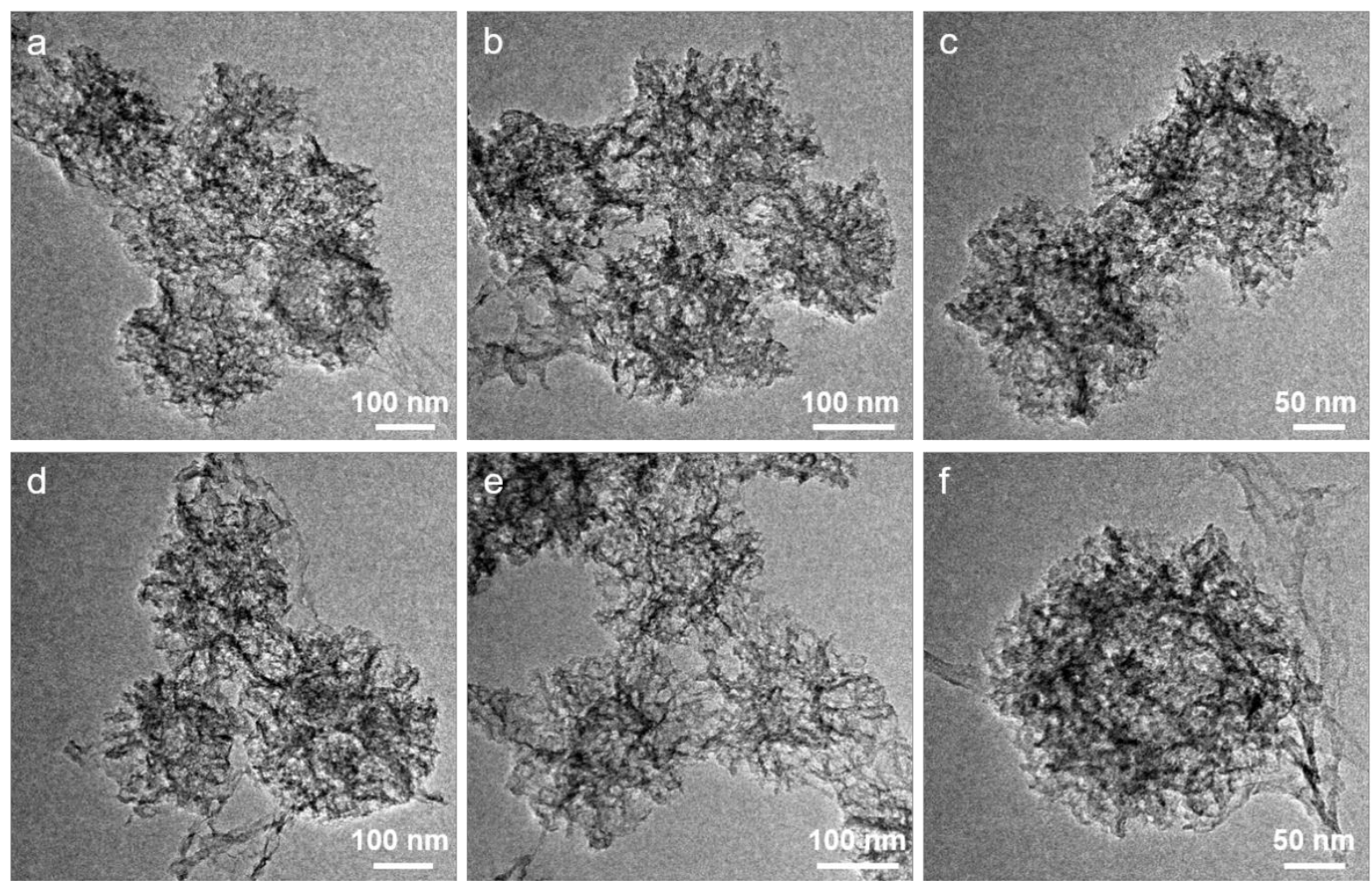

Figure S4. (a-f) TEM images at different magnifications of meso-NPC when the ratio of organics and meso- $\mathrm{SiO}_{2}$ sphere is $7.5: 1$. 

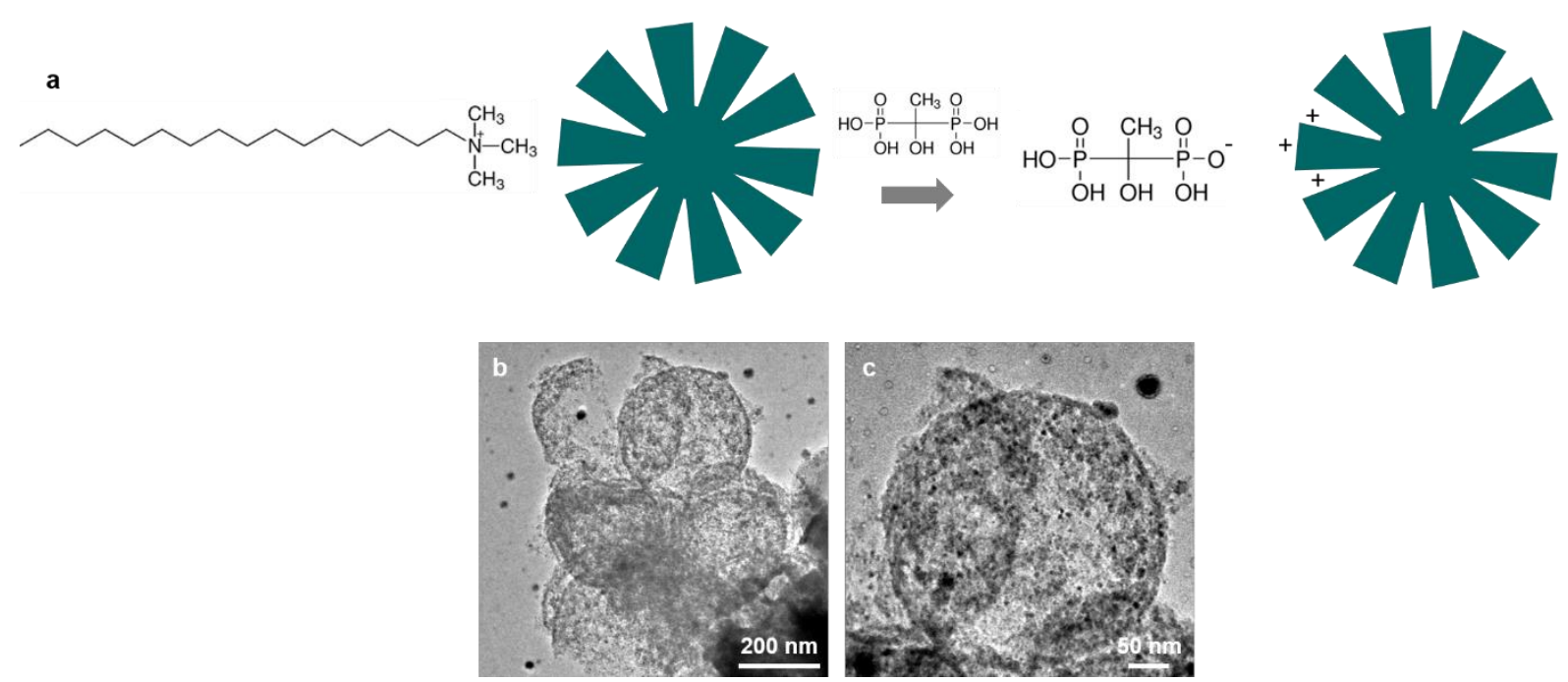

Figure S5 (a). Schematic illustration of the role of etidronic acid. It indicates the hydrogen ion can replace the original surfactant $\left(\mathrm{CTA}^{+}\right)$in the mesopores of $\mathrm{SiO}_{2}$, so that the organics could enter the mesopores. As a comparative study, when no etidronic acid was used, it leads to a product with no mesoporous channels as shown in the TEM images $(b, c)$.
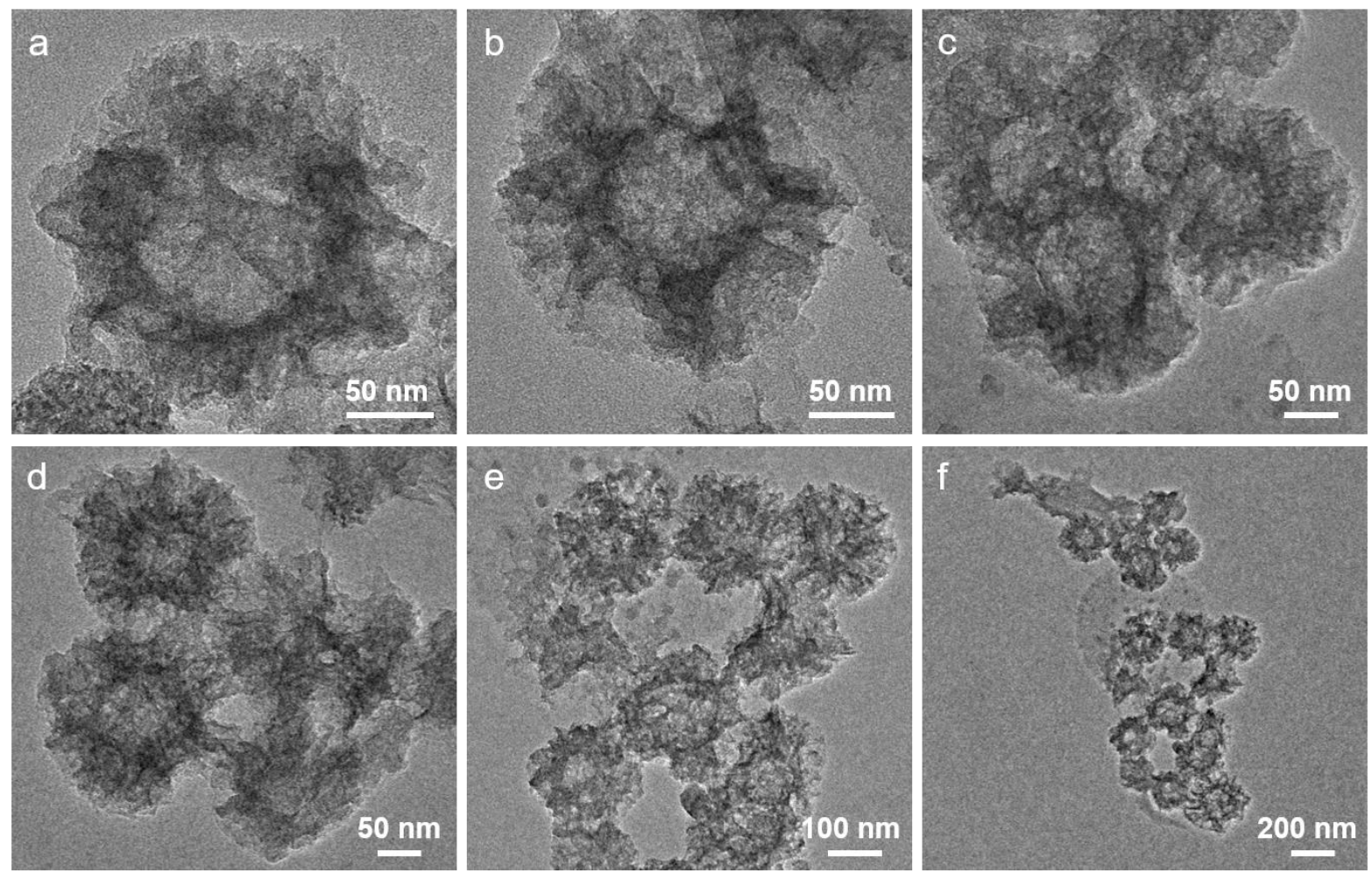

Figure S6. (a-f) TEM images at different magnifications of meso- $\mathrm{NP}_{1 / 2} \mathrm{C}$ sample. 

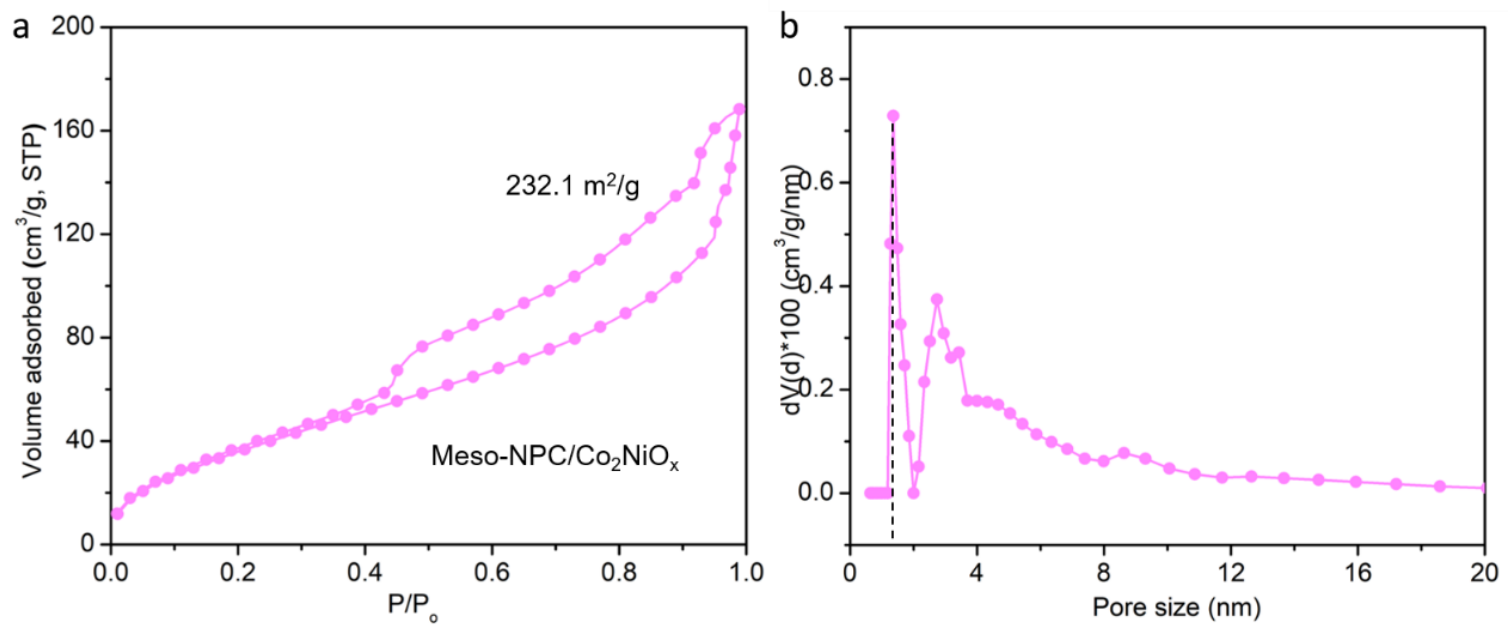

Figure S7. (a) $\mathrm{N}_{2}$ adsorption-desorption isotherm and (b) pore size distributions of meso$\mathrm{NPC} / \mathrm{Co}_{2} \mathrm{NiO}_{x}$ sample.

From the above results we can see, the specific surface area was further increased and abundant micropores at $1.36 \mathrm{~nm}$ were produced after loading ultrafine $\mathrm{Co}_{2} \mathrm{NiO}_{\mathrm{x}}$ nanoparticles. On the one hand, due to the small size and abundant hydroxyl groups, the meso-NPC hollow spheres are prone to aggregation after completely dry, this is the reason for meso-NPC have a much small specific surface area than expected. However, after loading $\mathrm{Co}_{2} \mathrm{NiO}_{\mathrm{x}}$ nanoparticles on the surface, the aggregation of meso-NPC can be effectively inhibited, which leads to a better exposition of the mesopores of meso-NPC. One the other hand, the production of micropores may be ascribed largely to the random stacks of the defective graphitized carbon sheets (Figure $2 \mathrm{f}$ of the main text) in the meso-NPC structure, which also contributes to the increase of total specific surface area. 


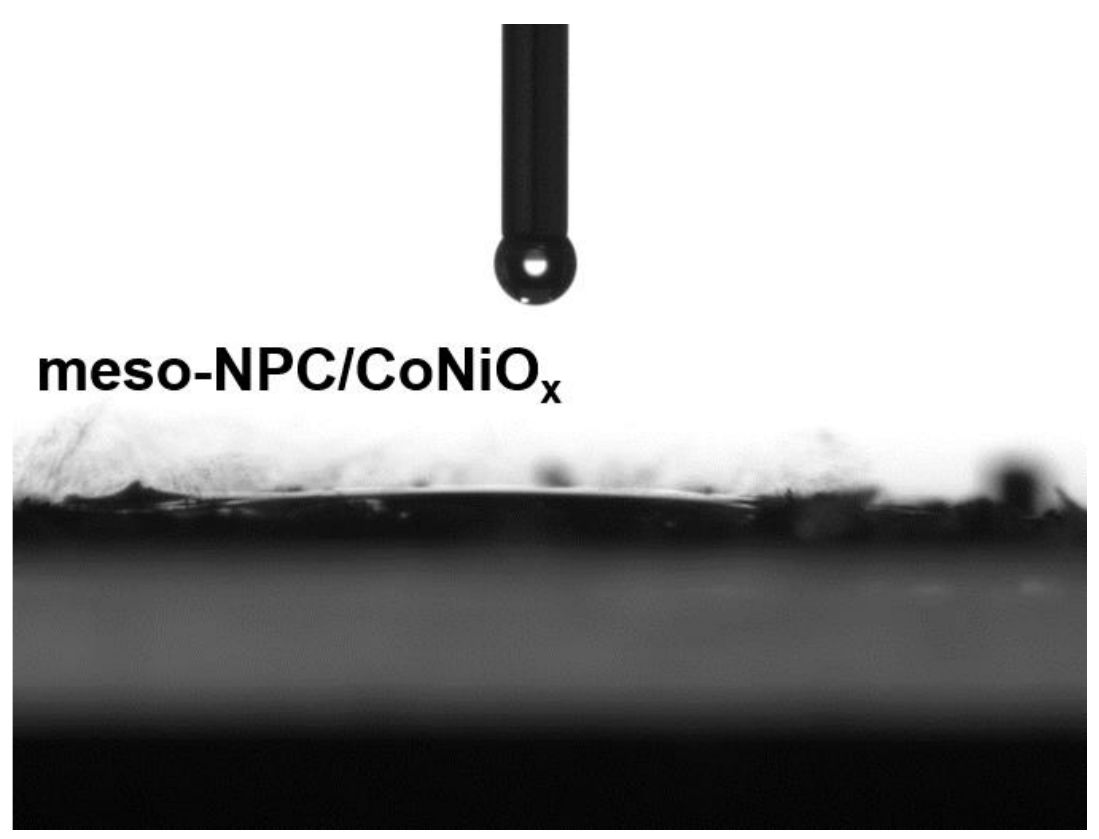

Figure S8. Photograph of meso-NPC/CoNiO sample substrate after loading a water droplet for 165 seconds.

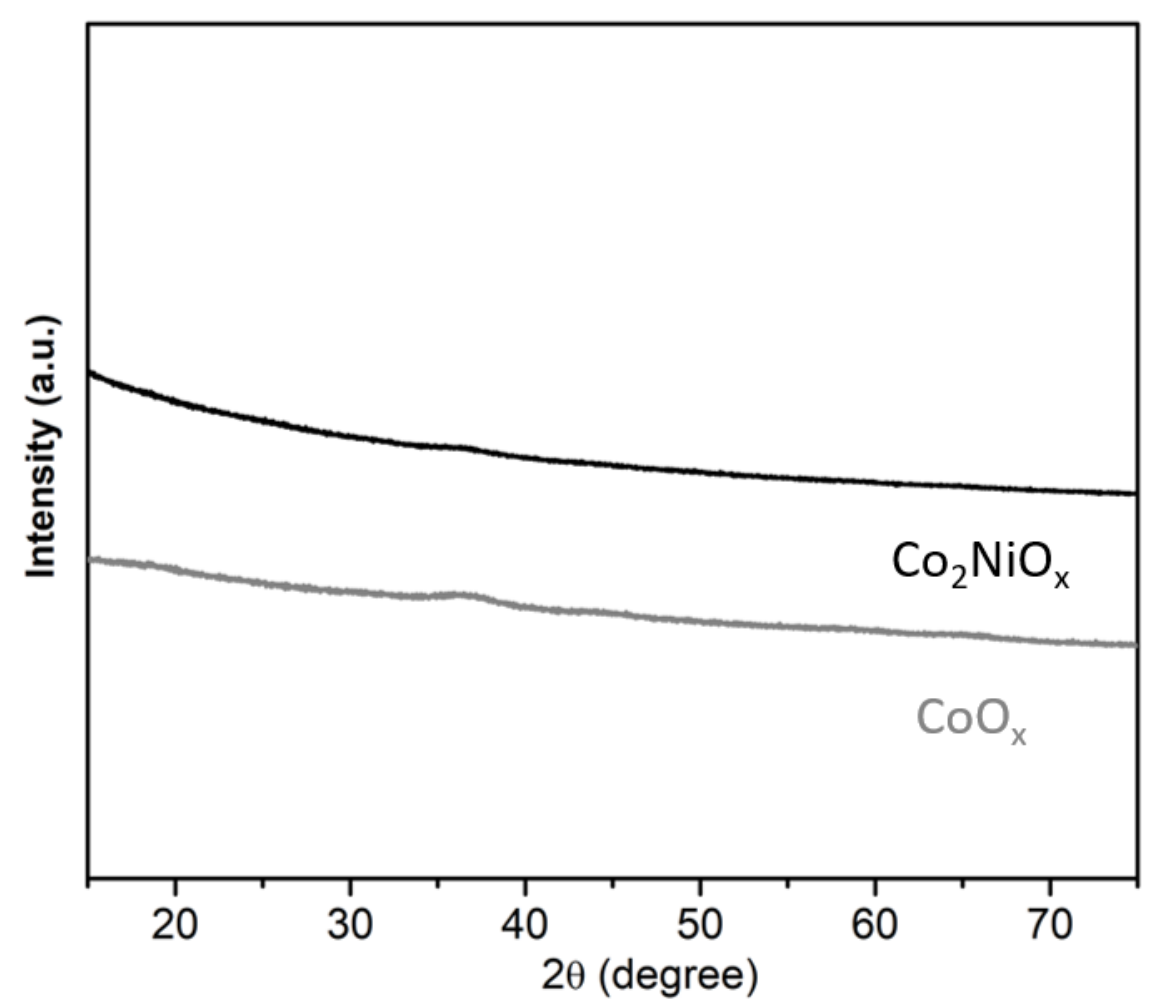

Figure S9. XRD patterns of $\mathrm{Co}_{2} \mathrm{NiO}_{\mathrm{x}}$ and $\mathrm{CoO}_{\mathrm{x}}$ samples. 


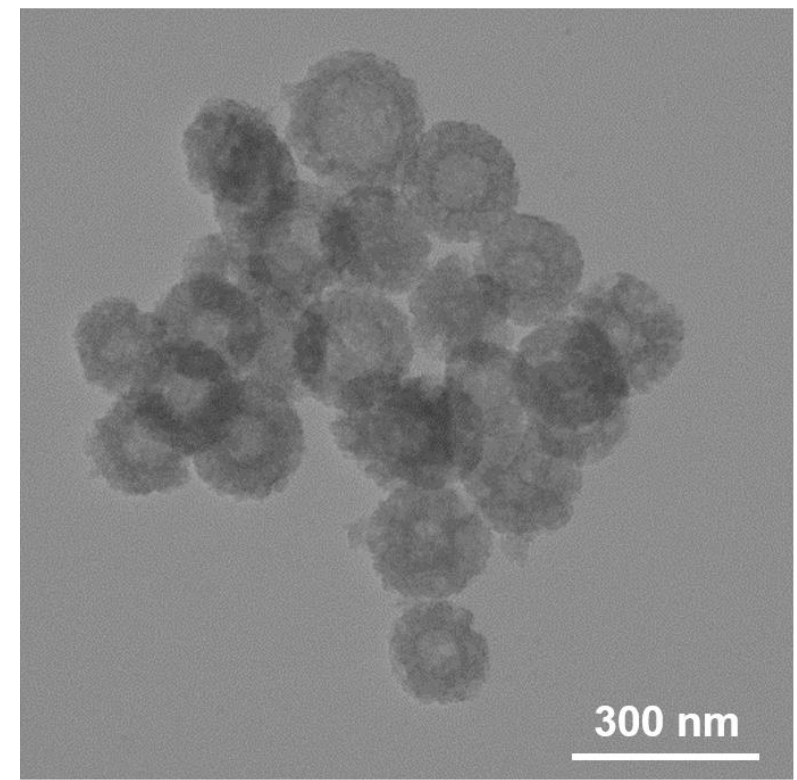

\begin{tabular}{|l|l|l|}
\hline Element & Weight\% & Atomic\% \\
\hline C K & 53.13 & 70.16 \\
\hline N K & 3.42 & 3.87 \\
\hline O K & 17.53 & 17.38 \\
\hline P K & 6.61 & 3.38 \\
\hline Co K & 12.92 & 3.48 \\
\hline Ni K & 6.38 & 1.72 \\
\hline Totals & 100.00 & \\
\hline
\end{tabular}

Figure S10. STEM image and EDX analytical data of meso-NPC/Co $2 \mathrm{NiO}_{x}$.

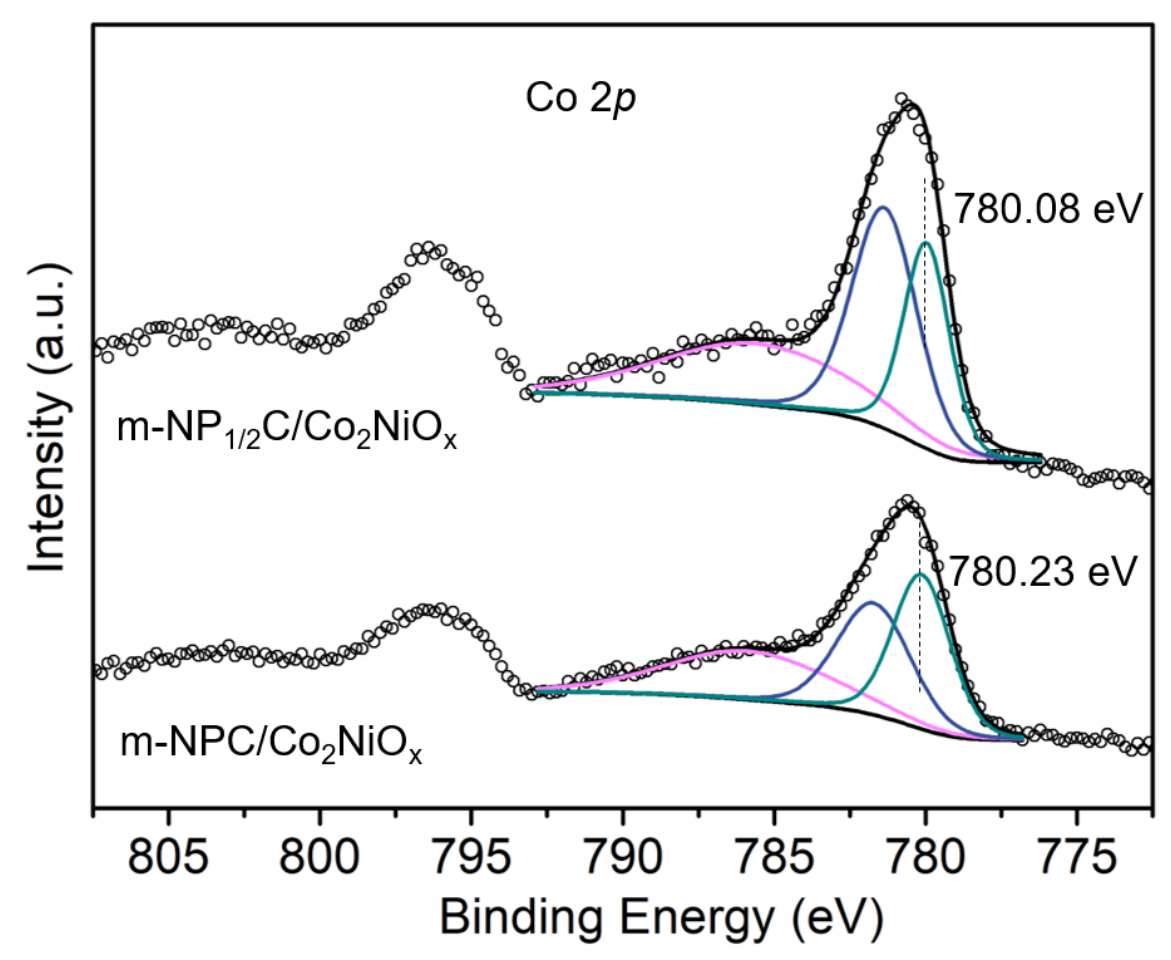

Figure S11. Comparison of high-resolution Co $2 p$ XPS spectra between meso$\mathrm{NP}_{1 / 2} \mathrm{C} / \mathrm{Co}_{2} \mathrm{NiO}_{x}$ and meso-NPC/Co2 $\mathrm{NiO}_{x}$. 

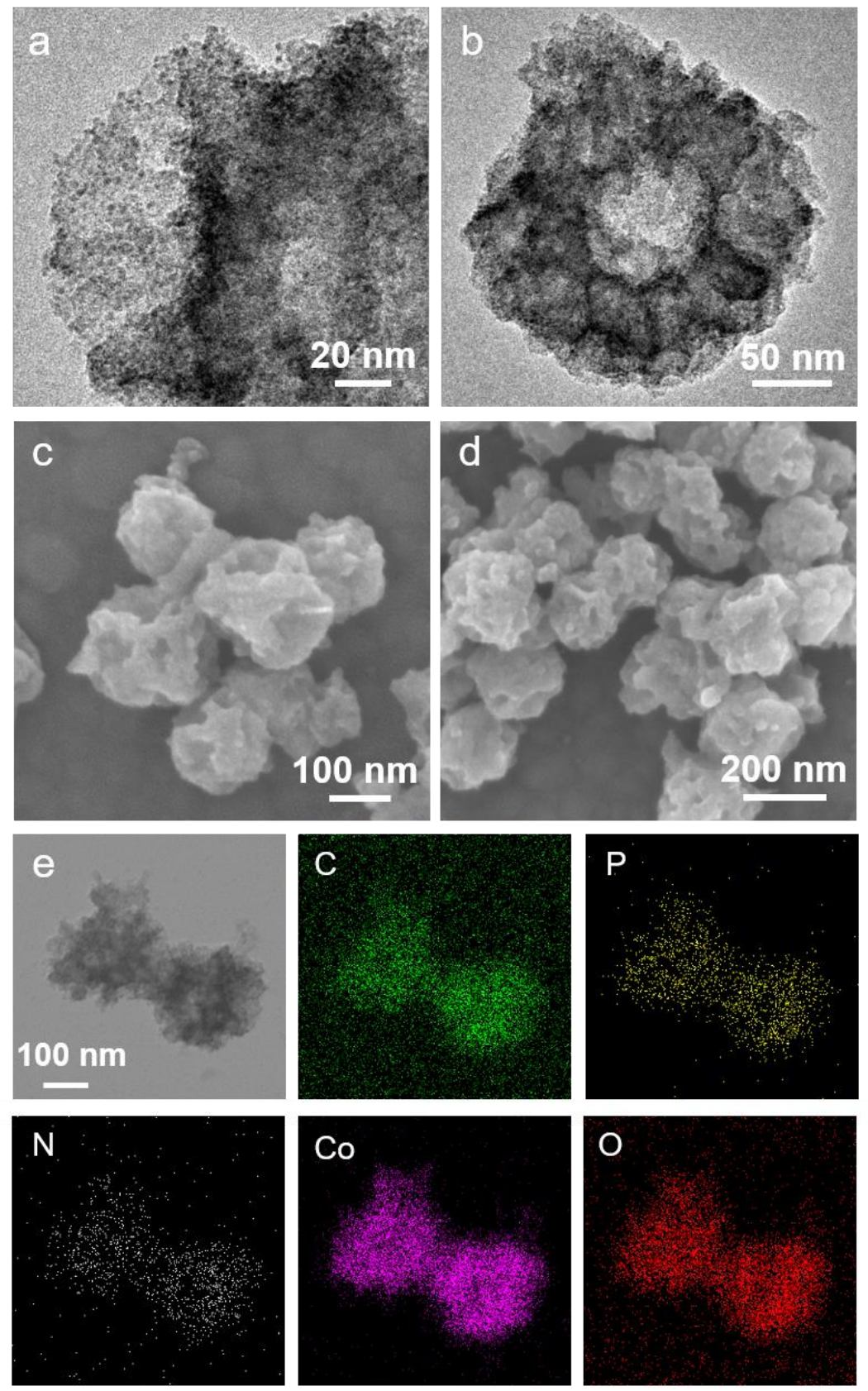

Figure S12. (a, b) TEM images and (c, d) SEM images and (e) Elemental mappings of meso$\mathrm{NPC} / \mathrm{CoO}_{\mathrm{x}}$. The preparation method of $\mathrm{CoO}_{x}$ colloid was similar with that of $\mathrm{Co}_{2} \mathrm{NiO}_{\mathrm{x}}$, just without the introduction of $\mathrm{Ni}$ precursor in the synthetic process. 

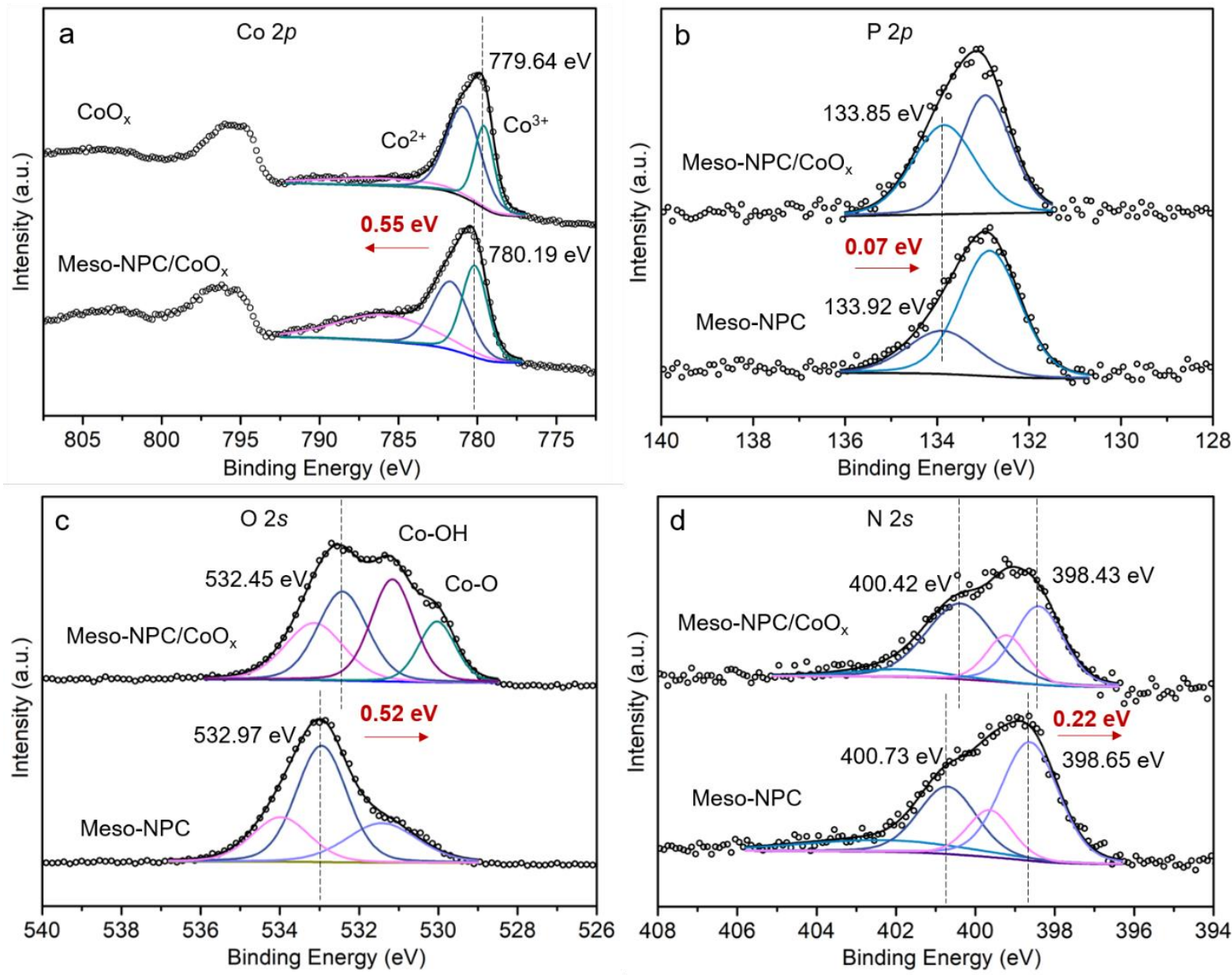

Figure S13. (a-d) Comparison of high-resolution XPS spectra among meso-NPC, $\mathrm{CoO}_{\mathrm{x}}$ and meso-NPC/CoO samples. 

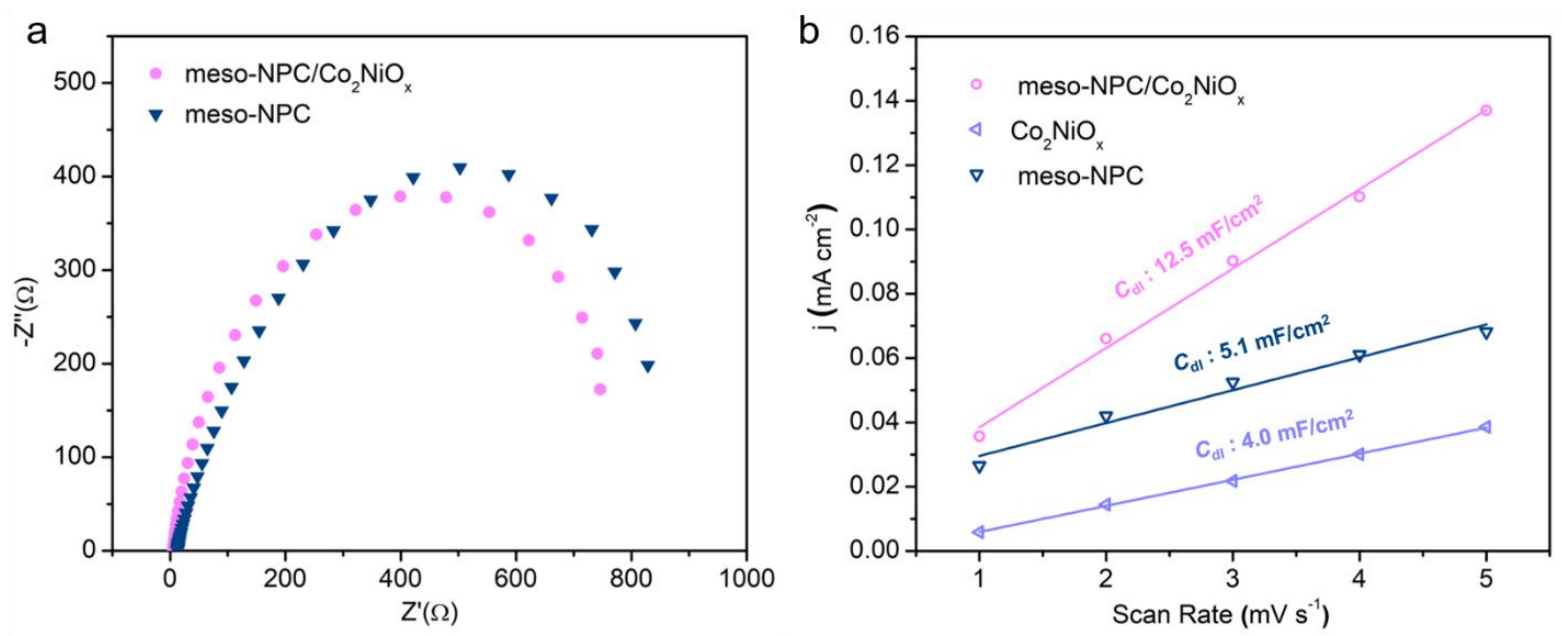

Figure S14. (a) The EIS results and (b) electrochemical active surface areas (ECSA) for some comparative samples.

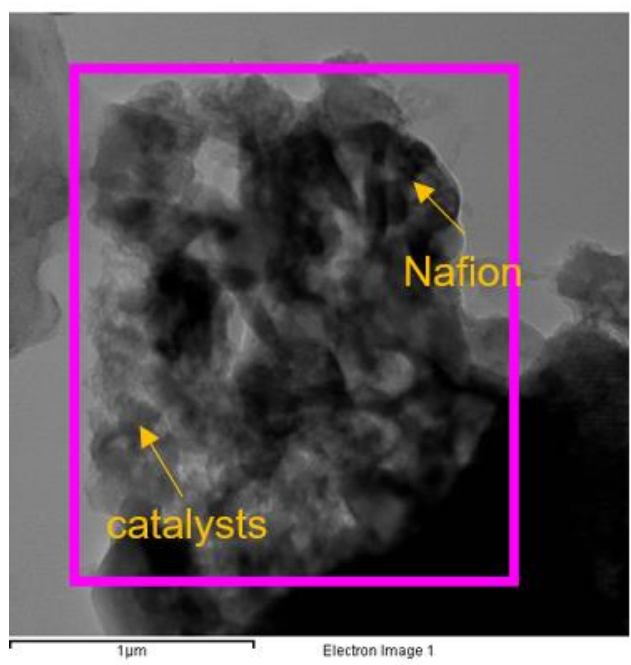

\begin{tabular}{|l|l|l|}
\hline Element & Weight\% & Atomic\% \\
\hline & & \\
\hline C K & 48.31 & 62.67 \\
\hline N K & 2.47 & 2.75 \\
\hline O K & 27.94 & 27.21 \\
\hline P K & 7.28 & 3.66 \\
\hline Co K & 9.61 & 2.54 \\
\hline Ni K & 4.39 & 1.16 \\
\hline & & \\
\hline Totals & 100.00 & \\
\hline
\end{tabular}

Figure S15. STEM image and EDX analytical data for the meso- $\mathrm{NPC} / \mathrm{Co} 2 \mathrm{NiO}_{x}$ after stability test. 
Table S1. The weight percentage of Co (and Ni) obtained from ICP-MS data for meso$\mathrm{NPC} / \mathrm{CoO}_{\mathrm{x}}$ and meso-NPC/Co $2 \mathrm{NiO}_{\mathrm{x}}$ samples.

\begin{tabular}{|c|c|c|}
\hline Sample & $\operatorname{Co}(w t \%)$ & $\mathrm{Ni}(w \mathrm{t} \%)$ \\
\hline Meso-NPC/CoO & 15.94 & 0 \\
\hline Meso-NPC/Co $2 \mathrm{NiO}_{x}$ & 10.52 & 5.27 \\
\hline
\end{tabular}

Table S2. Comparison of the OER catalytic performances of our currently prepared electrocatalysts to other recently reported high-performance OER electrocatalysts in alkaline solution.

\begin{tabular}{|c|c|c|c|c|c|c|}
\hline Catalyst & $\begin{array}{l}\text { Total loading } \\
\left(\mathrm{mg} \cdot \mathrm{cm}^{-2}\right)\end{array}$ & Electrolyte & Substrate & $\begin{array}{l}\eta(\mathrm{V}) @ 10 \\
\mathrm{~mA} \cdot \mathrm{cm}^{-2}\end{array}$ & $\begin{array}{l}\text { Tafel slope } \\
\left(\mathrm{mV} \cdot \mathrm{dec}^{-1}\right)\end{array}$ & $\begin{array}{l}\text { Ref. } \\
\text { (year) }\end{array}$ \\
\hline $\mathrm{NiCo}_{2} \mathrm{O}_{4}$ & 1.00 & $\begin{array}{l}1.0 \mathrm{M} \\
\mathrm{NaOH}\end{array}$ & NF & 0.29 & 53 & $2016^{1}$ \\
\hline NiFe-LDH/GO & 0.28 & $\begin{array}{l}1.0 \mathrm{M} \\
\mathrm{NaOH}\end{array}$ & GC & 0.21 & 52 & $2017^{2}$ \\
\hline $\begin{array}{l}\mathrm{NiCoP} / \mathrm{C} \\
\text { nanoboxes }\end{array}$ & 0.25 & $\begin{array}{l}1.0 \mathrm{M} \\
\mathrm{KOH}\end{array}$ & GC & 0.33 & 96 & $2017^{3}$ \\
\hline $\mathrm{N} / \mathrm{C}$ & 0.20 & $\begin{array}{l}0.1 \mathrm{M} \\
\mathrm{KOH}\end{array}$ & $\mathrm{GC}$ & 0.38 & & $2013^{4}$ \\
\hline $\begin{array}{l}\mathrm{NiCo}_{2} \mathrm{O}_{4} / \mathrm{N}- \\
\text { graphene }\end{array}$ & 0.20 & $\begin{array}{l}1.0 \mathrm{M} \\
\mathrm{KOH}\end{array}$ & GC & 0.31 & 88 & $2018^{5}$ \\
\hline $\mathrm{CoO}_{\mathrm{x}} \mathrm{NPs} / \mathrm{BNG}$ & & $\begin{array}{l}0.1 \mathrm{M} \\
\mathrm{KOH}\end{array}$ & $\mathrm{GC}$ & 0.30 & 57 & $2017^{6}$ \\
\hline $\begin{array}{l}\mathrm{ZnCo}_{2} \mathrm{O}_{4} / \mathrm{N}- \\
\text { doped-CNT }\end{array}$ & 0.20 & $\begin{array}{l}0.1 \mathrm{M} \\
\mathrm{KOH}\end{array}$ & $\mathrm{GC}$ & 0.42 & 70.6 & $2016^{7}$ \\
\hline NiFe@NC & 0.32 & $\begin{array}{l}1.0 \mathrm{M} \\
\mathrm{KOH}\end{array}$ & GC & 0.21 & 70 & $2016^{8}$ \\
\hline $\begin{array}{l}\text { Meso-NPC/ } \\
\mathrm{Co}_{2} \mathrm{NiO}_{x}\end{array}$ & 0.10 & $\begin{array}{l}1.0 \mathrm{M} \\
\mathrm{KOH}\end{array}$ & GC & 0.33 & 54 & $\begin{array}{l}\text { This } \\
\text { work }\end{array}$ \\
\hline
\end{tabular}




\section{REFERENCES}

1. Gao, X.; Zhang, H.; Li, Q.; Yu, X.; Hong, Z.; Zhang, X.; Liang, C.; Lin, Z., Hierarchical $\mathrm{NiCo}_{2} \mathrm{O}_{4}$ Hollow Microcuboids as Bifunctional Electrocatalysts for Overall Water-Splitting. Angew. Chem., Int. Ed. 2016, 55, 6290-6294.

2. Jia, Y.; Zhang, L.; Gao, G.; Chen, H.; Wang, B.; Zhou, J.; Soo, M. T.; Hong, M.; Yan, X.; Qian, G.; Zou, J.; Du, A.; Yao, X., A Heterostructure Coupling of Exfoliated Ni-Fe Hydroxide Nanosheet and Defective Graphene as a Bifunctional Electrocatalyst for Overall Water Splitting. Adv. Mater. 2017, 29, 0017-0024.

3. He, P.; Yu, X. Y.; Lou, X. W., Carbon-Incorporated Nickel-Cobalt Mixed Metal Phosphide Nanoboxes with Enhanced Electrocatalytic Activity for Oxygen Evolution. Angew. Chem., Int. Ed. 2017, 56, 3897-3900.

4. Zhao, Y.; Nakamura, R.; Kamiya, K.; Nakanishi, S.; Hashimoto, K., Nitrogen-doped carbon nanomaterials as non-metal electrocatalysts for water oxidation. Nat. Commun. 2013, 4, 23902397.

5. Wang, X. R.; Liu, J. Y.; Liu, Z. W.; Wang, W. C.; Luo, J.; Han, X. P.; Du, X. W.; Qiao, S. Z.; Yang, J., Identifying the Key Role of Pyridinic-N-Co Bonding in Synergistic Electrocatalysis for Reversible ORR/OER. Adv. Mater. 2018, 30, 0005-0014.

6. Tong, Y.; Chen, P.; Zhou, T.; Xu, K.; Chu, W.; Wu, C.; Xie, Y., A Bifunctional Hybrid Electrocatalyst for Oxygen Reduction and Evolution: Cobalt Oxide Nanoparticles Strongly Coupled to B,N-Decorated Graphene. Angew. Chem., Int. Ed. 2017, 56, 7121-7125.

7. Liu, Z. Q.; Cheng, H.; Li, N.; Ma, T. Y.; Su, Y. Z., ZnCo $\mathrm{O}_{4}$ Quantum Dots Anchored on Nitrogen-Doped Carbon Nanotubes as Reversible Oxygen Reduction/Evolution Electrocatalysts. Adv. Mater. 2016, 28, 3777-3784.

8. Cui, X.; Ren, P.; Deng, D.; Deng, J.; Bao, X., Single Layer Graphene Encapsulating Nonprecious Metals as High-performance Electrocatalysts for Water Oxidation. Energy Environ. Sci. 2016, 9, 123-129. 